\title{
SOME PROPERTIES OF A NEW SOLUTION OF THE ERNST EQUATION
}

\author{
J. Gariel ${ }^{1 *}$ G. Marcilhacy ${ }^{1}$ and N. O. Santos ${ }^{1,2,3 \dagger}$ \\ ${ }^{1}$ Université Pierre et Marie Curie - CNRS/UMR 8540, \\ LRM/ERGA, Tour 22-12, 4éme étage, Boîte 142, 4 place Jussieu, \\ 75252 Paris Cedex 05, France. \\ ${ }^{2}$ Laboratório Nacional de Computação Científica, \\ 25651-070 Petrópolis RJ, Brazil. \\ ${ }^{3}$ Centro Brasileiro de Pesquisas Físicas \\ 22290-180 Rio de Janeiro RJ, Brazil.
}

November 22, 2018

\begin{abstract}
From a particularly simple solution of the Ernst equation, we build a solution of the vacuum stationary axisymmetric Einstein equations depending on three parameters. The parameters are associated to the total mass of the source and its angular momentum. The third parameter produces a topological deformation of the ergosphere making it a two-sheet surface, and for some of its values forbids the Penrose process.
\end{abstract}

*e-mail: gariel@ccr.jussieu.fr

†e-mail: nos@cbpf.br 


\section{Introduction}

We propose a new solution of the Ernst equation. This solution is symmetric in the prolate spheroidal coordinates and depends on one parameter, called $q_{1}$. The construction of the corresponding gravitational potentials, with the help of the Boyer-Linquist transformation, shows that this solution has not a good asymptotical behaviour for its dragging. But, by an Ehlers transformation followed by an unitary transformation, which introduces two more parameters, an asymptotically flat solution can be easily built. So, the obtained solution, in Boyer-Linquist coordinates, depends on three parameters, which are connected, as in the Kerr case, to the total mass of the source and its angular momentum. However, we did not succeed to relate directly this solution to the Kerr solution, but we know that such a linck does exist by reason of the uniqueness theorem for the solution with a good asymptotical behaviour and without naked singularity [1]. Only the extreme black hole of Kerr appears as a limit of the proposed solution, and this latter does not present a naked singularity. Varying the $q_{1}$ parameter allows to show its role in the ergosphere shape. The ergosphere, which initially has a torus shape, continuously looses its form and finally separates into a two-sheet toroidal surface, progressively exposing more and more the event horizon. Then the Penrose process [2] is no longer able to take place in a domain of the azimuthal angle, for some range of the $q_{1}$ parameter values.

\section{Brief recall on the resolution of the Ernst equation}

The line element of a general axisymmetric stationary spacetime is the so called Papapetrou metric, which in the cylindrical coordinates, $\rho, z$ and $\phi$, reads

$$
d s^{2}=f(d t-\omega d \phi)^{2}-f^{-1}\left[e^{2 \gamma}\left(d \rho^{2}+d z^{2}\right)+\rho^{2} d \phi^{2}\right]
$$

where the gravitational potentials, $f, \omega$ and $\gamma$ are functions of $\rho$ and $z$ only. The canonical coordinates of Weyl, $\rho$ and $z$, can be given in terms of prolate spheroidal coordinates, $\lambda$ and $\mu$, by the relations

$$
\rho=k\left(\lambda^{2}-1\right)^{1 / 2}\left(1-\mu^{2}\right)^{1 / 2}, \quad z=k \lambda \mu
$$


with

$$
\lambda \geq 1, \quad-1 \leq \mu \leq 1, \quad k=\text { constant } .
$$

The metric (1) with relations (2) can be rewritten like

$$
\begin{aligned}
d s^{2}= & f(d t-\omega d \phi)^{2} \\
& -\frac{k^{2}}{f}\left[e^{2 \gamma}\left(\lambda^{2}-\mu^{2}\right)\left(\frac{d \lambda^{2}}{\lambda^{2}-1}+\frac{d \mu^{2}}{1-\mu^{2}}\right)+\left(\lambda^{2}-1\right)\left(1-\mu^{2}\right) d \phi^{2}\right]
\end{aligned}
$$

where the potentials are now functions of $\lambda$ and $\mu$. The Ernst equation is [3]

$$
(\xi \bar{\xi}-1) \nabla^{2} \xi=2 \bar{\xi} \nabla \xi \cdot \nabla \xi,
$$

where $\nabla$ and $\nabla^{2}$ are the gradient and the three-dimensional Laplacian operators respectively, $\bar{\xi}$ is the conjugated complex potential of $\xi$, and in general its solution can be expressed as

$$
\xi(\lambda, \mu)=P(\lambda, \mu)+i Q(\lambda, \mu),
$$

where $P$ and $Q$ are real functions of $\lambda$ and $\mu$. Among the classical solutions of the Ernst equation, we can cite the well known Kerr solution [3],

$$
\xi_{K}=p \lambda+i q \mu,
$$

where $p$ nd $q$ are real constants satisfying

$$
p^{2}+q^{2}=1 ;
$$

and the Tomimatsu-Sato solution,

$$
\xi_{T S}=\frac{\alpha(\lambda, \mu ; p, q, \delta)}{\beta(\lambda, \mu ; p, q, \delta)},
$$

where $\alpha$ and $\beta$ are two complex polynomials depending on the Kerr parameters $p$ and $q$ and a parameter $\delta$ assuming integer values describing the deformation of the source [四]. To determine the potentials $f, \omega$ and $\gamma$ of the metric (4), the method consists to use the following relation between $f$, the twist potential $\Phi$ and $\xi$,

$$
f+i \Phi=\frac{\xi-1}{\xi+1},
$$


which implies with (6),

$$
f=\frac{P^{2}+Q^{2}-1}{R^{2}}, \Phi=\frac{2 Q}{R^{2}}
$$

or, equivalently,

$$
f=1-\frac{\partial}{\partial P}\left[\ln \left(R^{2}+Q^{2}\right)\right], \quad \Phi=\frac{\partial}{\partial Q}\left[\ln \left(R^{2}+Q^{2}\right)\right],
$$

where

$$
R^{2}=(P+1)^{2}+Q^{2}
$$

In (10), $\Phi$ is the twist potential defined up to a constant and related to the dragging $\omega$ by the following differential equations,

$$
\frac{\partial \omega}{\partial \lambda}=\frac{k\left(1-\mu^{2}\right)}{f^{2}} \frac{\partial \Phi}{\partial \mu}, \quad \frac{\partial \omega}{\partial \mu}=-\frac{k\left(\lambda^{2}-1\right)}{f^{2}} \frac{\partial \Phi}{\partial \lambda} .
$$

The potential $\omega$ is obtained by integration of (14), and $\gamma$ is determined by quadratures. Any solution of the Ernst equation is a solution of the Einstein equations.

\section{A particular new solution}

It is easy to verify that a particular rational solution of the Ernst equation (5) is obtained when (6) has the following expressions for $P(\lambda, \mu)$ and $Q(\lambda, \mu)$,

$$
P=-q_{1}(\lambda+\mu), \quad Q=\frac{1+\lambda \mu}{\lambda+\mu}
$$

where $q_{1}$ is an arbitrary real parameter. In (15) we note a symmetry between $\lambda$ and $\mu$. However, it can be proved, it has not an axymptotically flat behaviour for the potentials $f$ and $\omega$. To obtain a flat asymptotical behaviour, a first step is to introduce a second real parameter, $\alpha_{1}$, by means of the following particular Ehlers transformation [5] on (15),

$$
\xi_{1}=\frac{c_{1} \xi+d_{1}}{\bar{d}_{1} \xi+\bar{c}_{1}}
$$


where

$$
c_{1}=1+i \alpha_{1}, \quad d_{1}=i \alpha_{1},
$$

satisfying

$$
\left(\begin{array}{cc}
c_{1} & d_{1} \\
\bar{d}_{1} & \bar{c}_{1}
\end{array}\right) \in S U(1,1), \quad\left|c_{1}\right|^{2}-\left|d_{1}\right|^{2}=1 .
$$

But, again, it can be proved, the solution (16) has still not the suitable asymptotical flatness. Then, a second step consists to perform an unitary transformation on $\xi_{1}$,

$$
\xi_{2}=e^{i \theta_{0}} \xi_{1}=(m+i n) \xi_{1}, m^{2}+n^{2}=1,
$$

with $\theta_{0}$ an arbitrary complex constant, and $m$ and $n$ real constants. Then (19) with (16) and (17) becomes

$\xi_{2}=\frac{P\left(m-\alpha_{1} n\right)-Q\left(\alpha_{1} m+n\right)-\alpha_{1} n+i\left[P\left(\alpha_{1} m+n\right)+Q\left(m-\alpha_{1} n\right)+\alpha_{1} m\right]}{-\left(\alpha_{1} Q+1\right)+i \alpha_{1}(P+1)}$.

Considering

$$
\alpha_{1}=-\frac{n}{2(1+m)}
$$

and applying the method recalled in section 2, we find the potentials corresponding to the solution (20) of the Ernst equation

$$
\begin{aligned}
f & =\left\{\frac{(1+\lambda \mu)^{2}+(\lambda+\mu)^{2}\left[q_{1}^{2}(\lambda+\mu)^{2}-1\right]}{\left.(1+\lambda \mu)^{2}+(\lambda+\mu)^{2}\left[q_{1}(\lambda+\mu)+1\right]^{2}\right]}\right\} \cos ^{-2} \frac{\theta_{0}}{2}, \\
\Phi & =-2\left\{\frac{(1+\lambda \mu)(\lambda+\mu)}{(1+\lambda \mu)^{2}+(\lambda+\mu)^{2}\left[q_{1}(\lambda+\mu)+1\right]^{2}}\right\} \cos ^{-2} \frac{\theta_{0}}{2}, \\
\omega & =\frac{2 k}{q_{1}}\left\{\frac{\left(1-\mu^{2}\right)\left(\lambda^{2}-1\right)\left[1+q_{1}(\lambda+\mu)\right]}{(1+\lambda \mu)^{2}+(\lambda+\mu)^{2}\left[q_{1}^{2}(\lambda+\mu)^{2}-1\right]}\right\} \cos ^{2} \frac{\theta_{0}}{2} .
\end{aligned}
$$

More, from (20) with (19), we find for $\gamma$ in (1),

$$
\gamma=\frac{1}{2} \ln \left[q_{1}^{2}-\frac{\left(\lambda^{2}-1\right)\left(1-\mu^{2}\right)}{(\lambda+\mu)^{4}}\right] .
$$

Furthermore, the factor $\cos ^{-2}\left(\theta_{0} / 2\right)$ in (22) can be absorbed by a rescaling process of the metric into a conformal metric, like $d s_{2}^{2}=\cos ^{2}\left(\theta_{0} / 2\right) d s^{2}$. 
Now introducing spherical coordinates $r$ and $\theta$, through the Boyer-Lindquist transformation [6],

$$
\lambda=\frac{r-M}{k}, \quad \mu=\cos \theta
$$

into (22) and (24), we obtain asymptotically $r \rightarrow \infty$,

$$
\begin{aligned}
& f \approx 1-2 \frac{k}{q_{1}} \frac{1}{r}+O\left(\frac{1}{r^{2}}\right), \\
& \omega \approx 2\left(\frac{k}{q_{1}}\right)^{2} \cos ^{2} \frac{\theta_{0}}{2} \frac{1-\mu^{2}}{r}+O\left(\frac{1}{r^{2}}\right) .
\end{aligned}
$$

We see from (27) and (28) that the solution now has the good asymptotic behaviour allowing us to interpret the parameters $q_{1}, \theta_{0}$ and $k$ as

$$
\frac{k}{q_{1}}=M, \quad \cos ^{2} \frac{\theta_{0}}{2}=\frac{J}{M^{2}}=\frac{a}{M},
$$

where $M$ and $J$ are, respectively, the mass and the angular momentum of the source, and $a=J / M$ the angular momentum per unit mass. In the Kerr solution (7) there are two parameters linked by the condition (8). The asymptotical behaviour of this solution imposes [7]

$$
p=\frac{k}{M}, \quad q=\frac{a}{M}
$$

and the condition (8) fixes $k$,

$$
k^{2}=M^{2}-a^{2} .
$$

In our solution, the asymptotical relations (27) and (28) impose (29), but $q_{1}$, $\theta_{0}$ and $k$ are arbitrary, as can be seen from (15), (19) and (26). Of course, it is always possible to compare our parameters to those of Kerr by putting

$$
\frac{a}{M}=q=\cos ^{2} \frac{\theta_{0}}{2}, \frac{k}{M}=p=q_{1},
$$

and assuming $0 \leq q_{1} \leq 1$. So, we would have also from (8),

$$
q_{1}^{2}+\cos ^{4} \frac{\theta_{0}}{2}=1 .
$$


However, it is not necessary for us to choose (32) and (33). In general, our

solution presents three independent free parameters, $q_{1}, \theta_{0}$ and $k$, whereas the Kerr solution presents only one independent parameter, either $p$ or $q$. Furthermore, imposing (32) and (33), does not reduce our solution to the Kerr solution. The differences between both solutions are further studied in the next section. Besides, we note that the solution (22)-(24) does not belong to the usual Tomimatsu-Sato solutions [4].

\section{Horizons, ergospheres and singularities}

The expression (22) can be written like

$$
f=\frac{N}{D} \cos ^{-2} \frac{\theta_{0}}{2}
$$

with

$$
\begin{gathered}
N=(1+\lambda \mu)^{2}+(\lambda+\mu)^{2}\left[q_{1}^{2}(\lambda+\mu)^{2}-1\right], \\
D=(1+\lambda \mu)^{2}+(\lambda+\mu)^{2}\left[q_{1}(\lambda+\mu)+1\right]^{2} .
\end{gathered}
$$

\subsection{Horizons}

The horizons correspond to the solution of $f=0$ for $\mu= \pm 1$ which is, from (35), $\lambda=\mp 1$, or from (26), $r_{h}=M \pm k$. These horizons split into the Cauchy horizon, with radius $r_{c h}=M-k$, and the event horizon, with radius $r_{e h}=M+k$. These results are satisfactory since, for any stationary axisymmetric metric, the horizons depend only on the spacetime symmetries.

\subsection{Ergospheres}

The equation of the ergosphere surfaces, from (34), is $N=0$, and two cases have to be distinguished.

\subsection{1 $\lambda+\mu=0$}

In this case $N=0$ if, in addition,

$$
1+\lambda \mu=0
$$


which imposes two solutions, describing two points,

$$
\begin{aligned}
& \lambda=-1, \quad \mu=1, \\
& \lambda=1, \quad \mu=-1 .
\end{aligned}
$$

These points, (38) and (39), are the intersections of the $z$ axis with the event horizon, $r=r_{e h}=M+k$, and Cauchy horizon, $r=r_{c h}=M-k$, respectively, belonging to the ergospheres. It has to be noted that (38) and (39) produce, from (36), $D=0$ as well, hence there is an indetermination for the ratio $N / D$. This indetermination can be raised by studying the limits $\lambda \rightarrow \pm 1$ which produce

$$
\lim _{\lambda \rightarrow-1} f(\mu=1)=\lim _{\lambda \rightarrow 1} f(\mu=-1)=0 .
$$

The limits (40) are finite and zero, hence these points belong to the ergospheres.

\subsection{2 $\lambda+\mu \neq 0$}

In this case, $(\lambda+\mu)^{2}$ can be factorized in (35), and the equation for $N=0$ becomes

$$
\left(\frac{1+\lambda \mu}{\lambda+\mu}\right)^{2}+q_{1}^{2}(\lambda+\mu)^{2}=1,
$$

which is the equation for the ergosphere surfaces. It is a fourth degree surface, and for the representation of this surface, it is useful to express it through a parametric representation with the help of a parameter $\tau$, such that,

$$
\left(\frac{1+\lambda \mu}{\lambda+\mu}\right)^{2}=\cos ^{2} \tau, \quad q_{1}^{2}(\lambda+\mu)^{2}=\sin ^{2} \tau .
$$

We can see from (42) that it is a bounded closed surface for any value of the $q_{1}$ parameter. We have plotted some curves, which are intersections of this surface by the meridian plane $\phi=0$, for different values of the parameter $1 \geq q_{1}>0$, as shown in figs. 1-10. These curves present the following interesting features.

- When $q_{1} \rightarrow 0$ the aspect of the ergospheres and horizons tends towards the aspect of the Kerr extreme black hole (e.g. see fig.4 of [8]), as shown in fig.1. 
- When $q_{1}$ increases its value the aspect of the ergospheres remarkably differs from this of a Kerr black hole, as shown in figs. 2-5. Specially, we can notice, the surface of the exterior ergosphere becomes double, presenting some thickness being a two-sheet torus. It is the same for the interior ergosphere.

- For a defined value of $q_{1}$, near $q_{1} \approx 0.5$, the exterior ergosphere opens itself on the axis $\mu=0(\theta=\pi / 2)$, as shown in figs. 6-8. Then the event-horizon becomes naked in a certain angular aperture, whereas the Kerr event-horizon is always dressed by the exterior surface of the ergosphere. Thus, on this spatial portion, the Penrose process [2] is no longer able to take place. This special topology of the ergosphere indicates, also here, a difference with the Kerr metric.

- The evolution of the interior part of the ergosphere, for increasing values of $q_{1}$, looks intricate, with, particularly, the advent from the center of a new curve, as shown in figs. 3-4, with a four-leaved clover shape, which grows up until to pass beyond the Cauchy horizon, as shown in figs. 8-9, which of course vanishes when $q_{1}=1(M=k)$, as shown in fig. 10. This complicated behaviour presents, also here, an important difference with the Kerr metric, because in this last case, the Cauchy horizon always covers the interior ergosphere.

Figs. 1-10 show the parametric plots of the curves describing the intersections of the interior and exterior ergospheres,defined by eq.(4.1), with the meridian plane $\phi=0$ for different values of the parameter $q_{1}$ in the range $\left[10^{-2}, 1\right]$. The vertical axis is z. The ergospheres are the axisymmetric surfaces which can be generated by rotation of the curves around the z-axis. The event-horizon and Cauchy-horizon are also represented (circles of radius $r_{e h}=M+k, r_{c h}=M-k$, respectively). The mass $M$ has been fixed to the value $M=4$. $k$ is given by eq. (29).

\subsection{Singularities}

The singularities correspond, when they exist, to curves or surfaces defined by $D=0$ from (36). We see that $D$ is a sum of squares and it can vanish only in two cases: 


\subsection{1 $1+\lambda \mu=0$ and $\lambda+\mu=0$}

This system of equations is the same as studied in 4.2.1, and corresponds to the two points (38) and (39) of the horizons where $N=0$. Since, after raising the indetermination of the ratio $N / D$, the limit (40) is finite and zero, these two points are not singular.

\subsection{2 $1+\lambda \mu=0$ and $q_{1}(\lambda+\mu)=-1$}

Or, equivalently,

$$
\begin{array}{r}
\lambda=-\frac{1}{\mu}, \\
q_{1} \mu^{2}+\mu-q_{1}=0 .
\end{array}
$$

The polynomial (44) always has two roots,

$$
\mu_{ \pm}=\frac{-1 \pm \sqrt{\Delta}}{2 q_{1}}, \quad \Delta=1+4 q_{1}^{2},
$$

that gives the two solutions,

$$
\mu_{+}=\frac{-1+\sqrt{\Delta}}{2 q_{1}}, \quad \lambda_{+}=-\frac{1}{\mu_{+}}
$$

and

$$
\mu_{-}=-\frac{1+\sqrt{\Delta}}{2 q_{1}}, \quad \lambda_{-}=-\frac{1}{\mu_{-}} .
$$

The first solution, (46), produces $0 \leq \mu_{+} \leq 1$, while the second, (47), produces $\left|\mu_{-}\right|>1$, hence it has to be rejected. From (46) with (26), we have

$$
r_{+}=M\left(1+\frac{2 q_{1}^{2}}{1-\sqrt{\Delta}}\right)
$$

which gives $r_{+}<r_{c h}=M-k$, hence the two ring singularities (48), which are the solutions for $\mu_{+}=\cos \left( \pm \theta_{+}\right)$, are inside the Cauchy horizon and so, a fortiori, inside the event horizon. There are no naked singularities. 


\section{Conclusion}

It has been proposed a new axially symmetrical stationary vacuum solution (15) of Ernst equation. Unfortunately, this solution does not satisfy the aymptotical flatness. Only after performing an Ehlers transformation and an unitary transformation, the solution (22)-(24) achieves the appropriate physical asymptotical flatness. Three arbitrary parameters were introduced in this process and interpreted from the asymptotical properties of the solution related to the total mass of its source and its angular momentum. We did not succeed to obtain the Kerr limit of the solution, however we know that it does exist because of the uniqueness theorem, since this new solution has asymptotical flatness and does not present naked singularities. One of the parameters introduced shapes the ergosphere demonstrating big differences to the Kerr solution. When this parameter vanishes the solution becomes the extreme Kerr black hole. We might conjecture that the solution (22)-(24) represents a distorted stationary black hole as obtained in the static case [9].

\section{Acknowledgments}

The authors wish to thank R.Colistete Jr. for his valuable help discussing and formatting this article.

\section{References}

[1] Carter B 1971 Phys. Rev. Lett. 26, 331; Robinson D C 1975 Phys. Rev. Letters 34, 905.

[2] Penrose R and Floyd R M 1971 Nature Phys. Sci. 229, 177.

[3] Ernst F J 1968 Phys. Rev. 167, 1175.

[4] Tomimatsu A and Sato H 1972 Phys. Rev. Lett. 29, 1344; 1973 Prog. Theor. Phys. 50, 95.

[5] Chandrasekhar S 1983 The Mathematical Theory of Black Holes (Oxford University Press, Oxford) p. 285. 
[6] Carmeli M 1982 Classical Fields: General Relativity and Gauge Theory (John Wiley and Sons, New York) p. 387.

[7] Kramer D, Stephani H, Herlt E and MacCallum M 1980 Exact Solutions of Einstein's Field Equations (Cambridge University Press, Cambridge) p. 206.

[8] Gariel J, Marcilhacy G, Santos NO and Colistete R (2000).Parametrization of singularities of the Demiamski-Newman spacetimes, grqc/0012004.

[9] Geroch R and Hartle J B 1982 J. Math. Phys. 23, 680. 


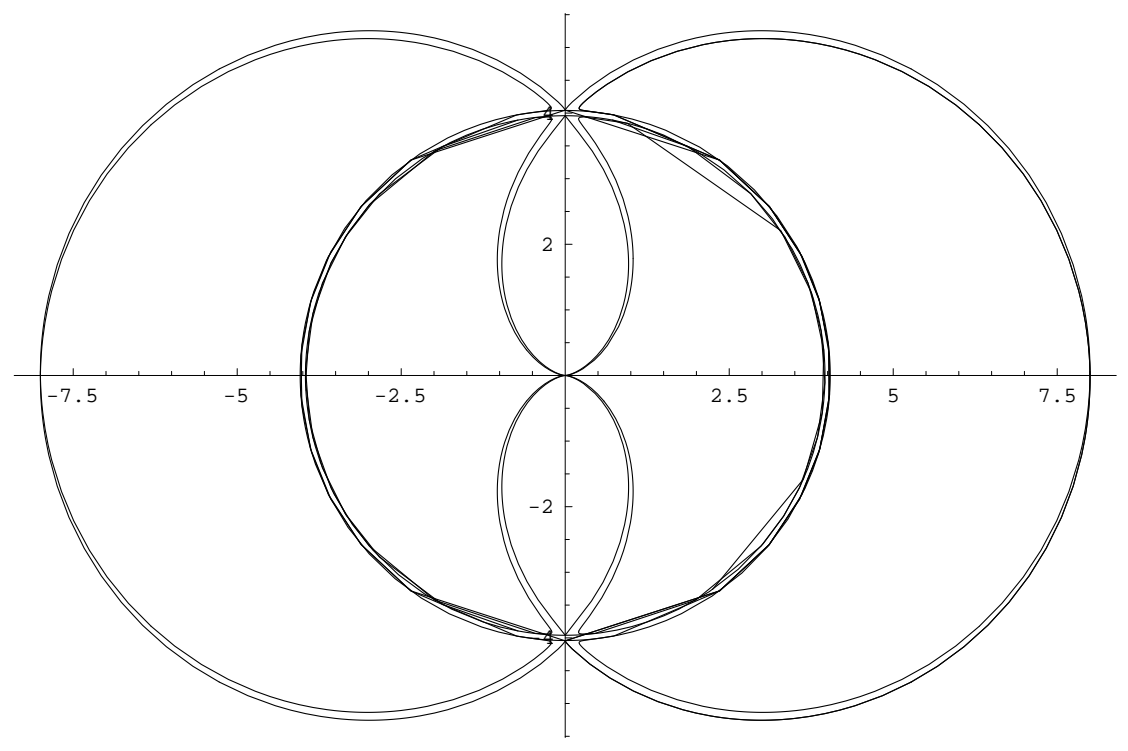

Figure 1: $q_{1}=0.01, r_{e h}=4.04, r_{c h}=3.96$.

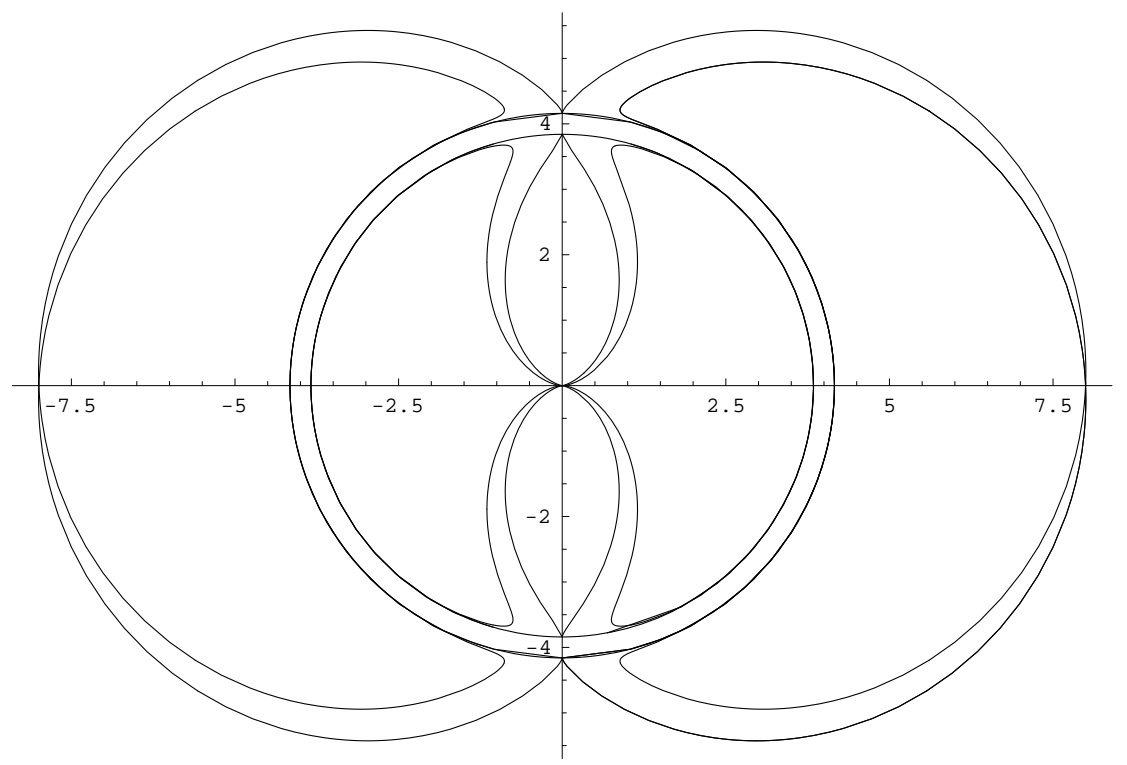

Figure 2: $q_{1}=0.04, r_{e h}=4.16, r_{c h}=3.84$. 


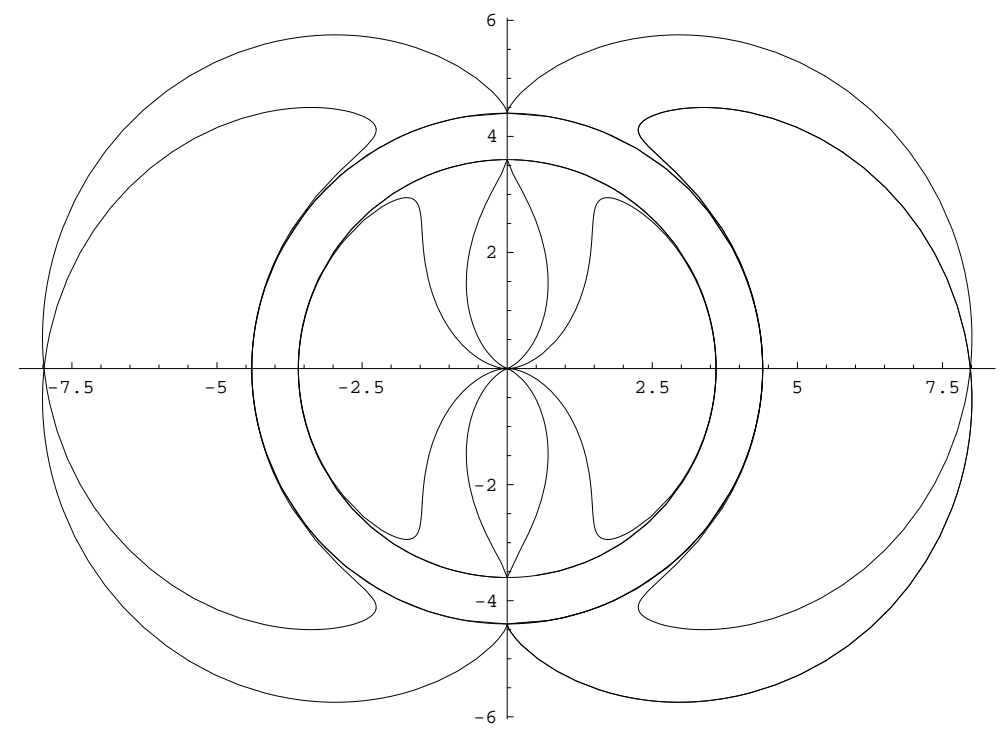

Figure 3: $q_{1}=0.1, r_{e h}=4.4, r_{c h}=3.6$.

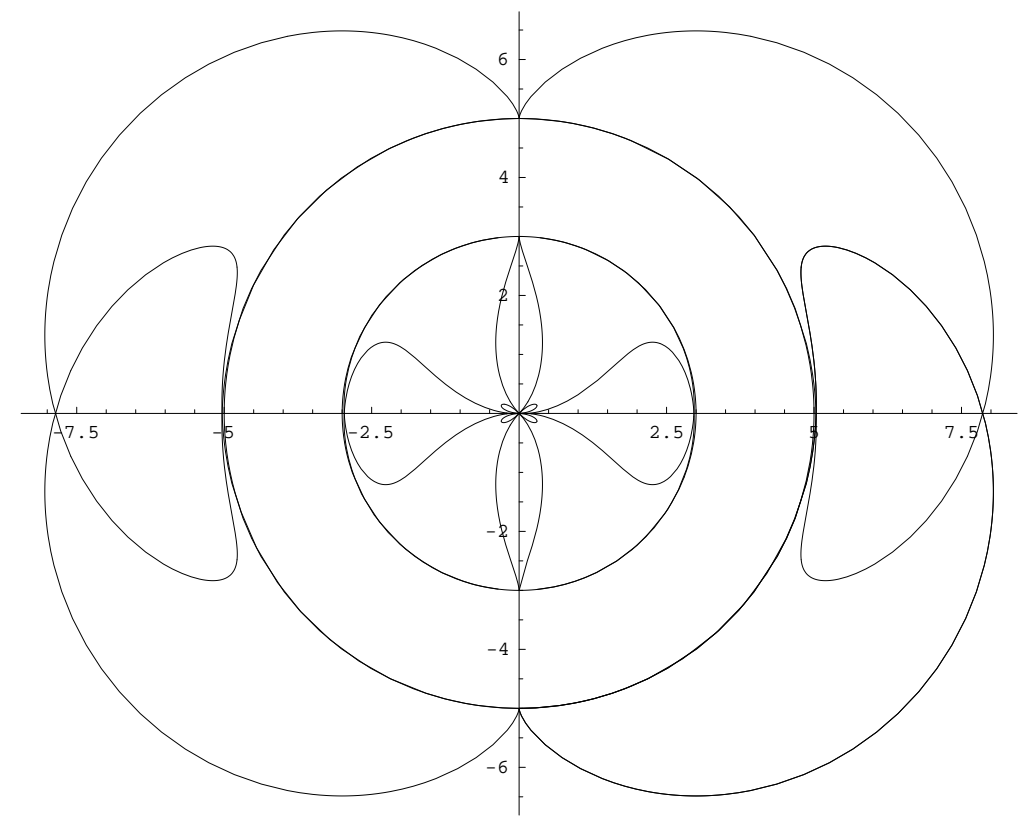

Figure 4: $q_{1}=0.25, r_{e h}=5, r_{c h}=3$. 


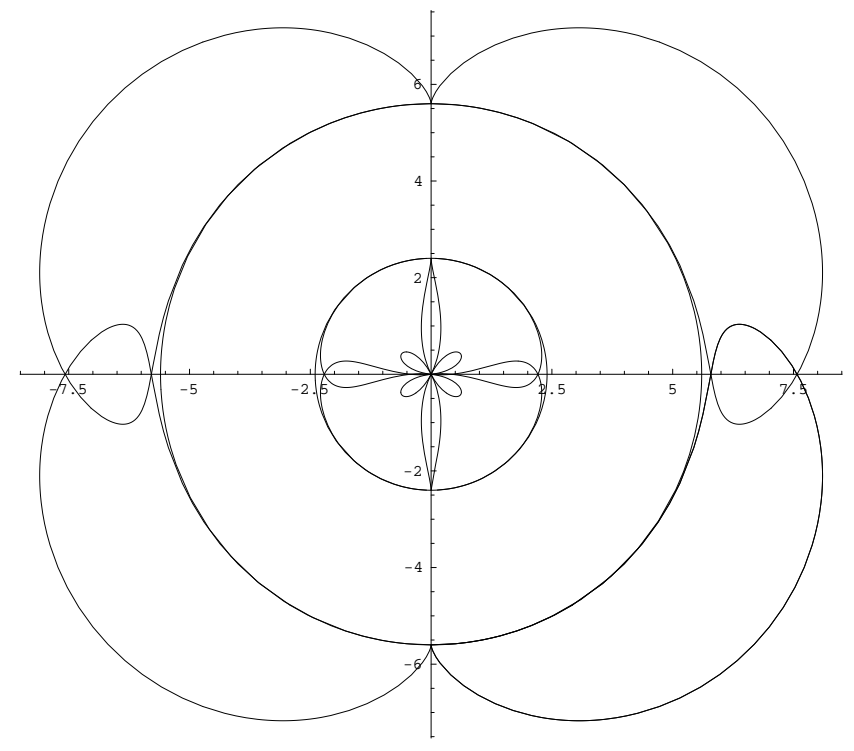

Figure 5: $q_{1}=0.4, r_{e h}=5.6, r_{c h}=2.4$.

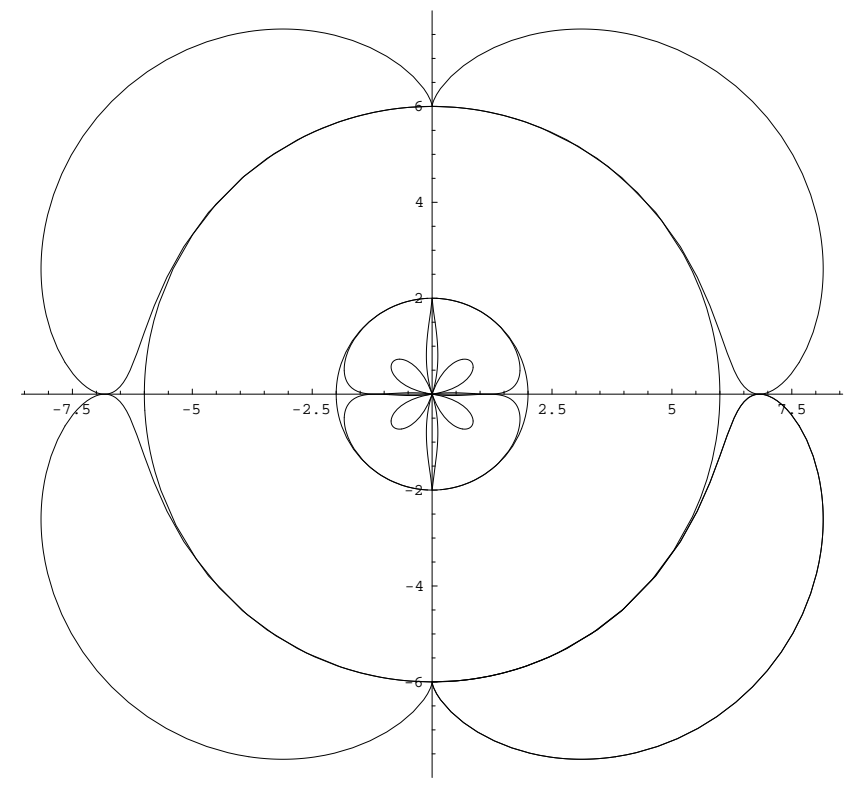

Figure 6: $q_{1}=0.5, r_{e h}=6, r_{c h}=2$. 


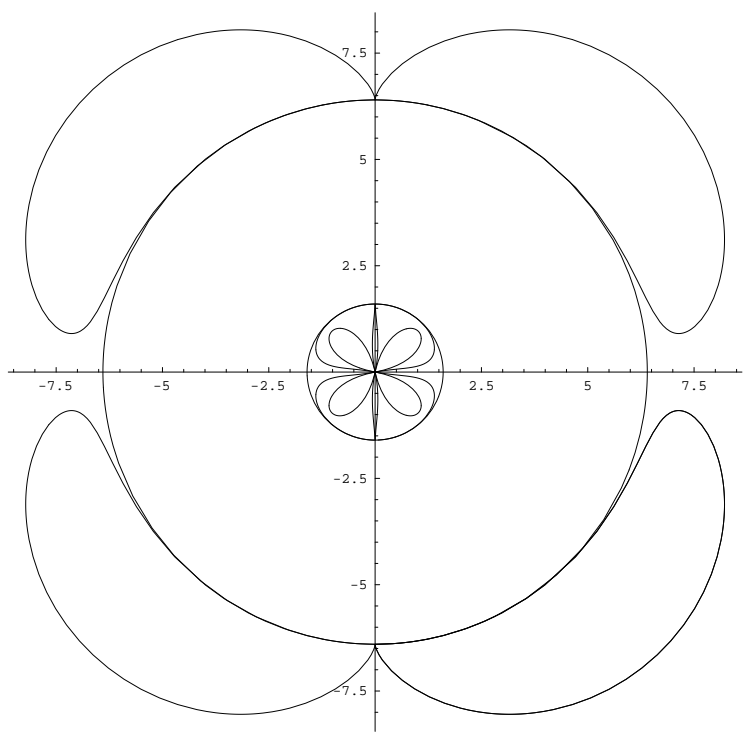

Figure 7: $q_{1}=0.6, r_{e h}=6.4, r_{c h}=1.6$.

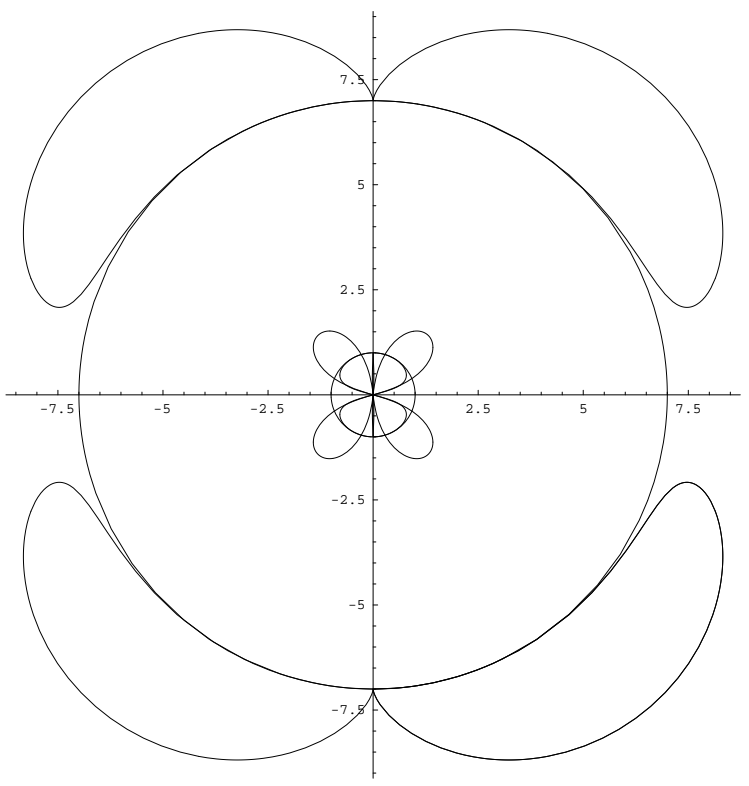

Figure 8: $q_{1}=0.75, r_{e h}=7, r_{c h}=1$. 


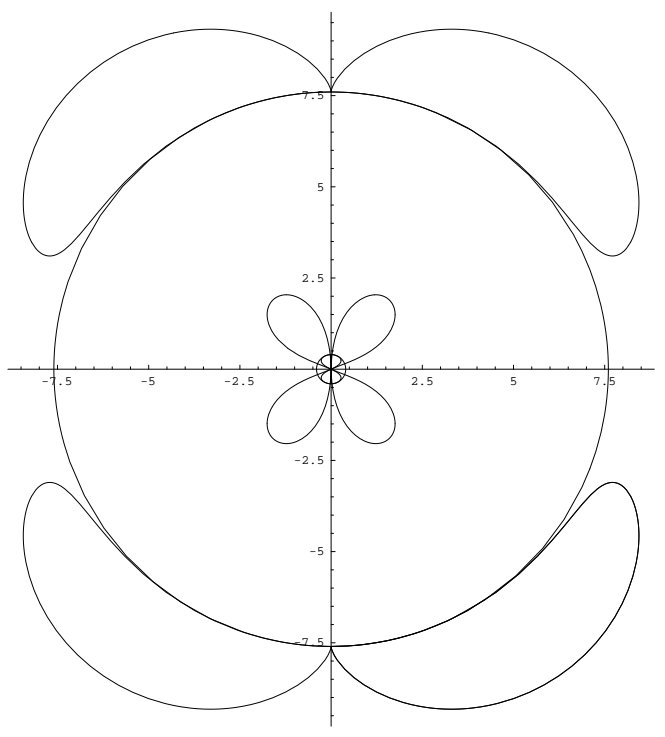

Figure 9: $q_{1}=0.9, r_{e h}=7.6, r_{c h}=0.4$.

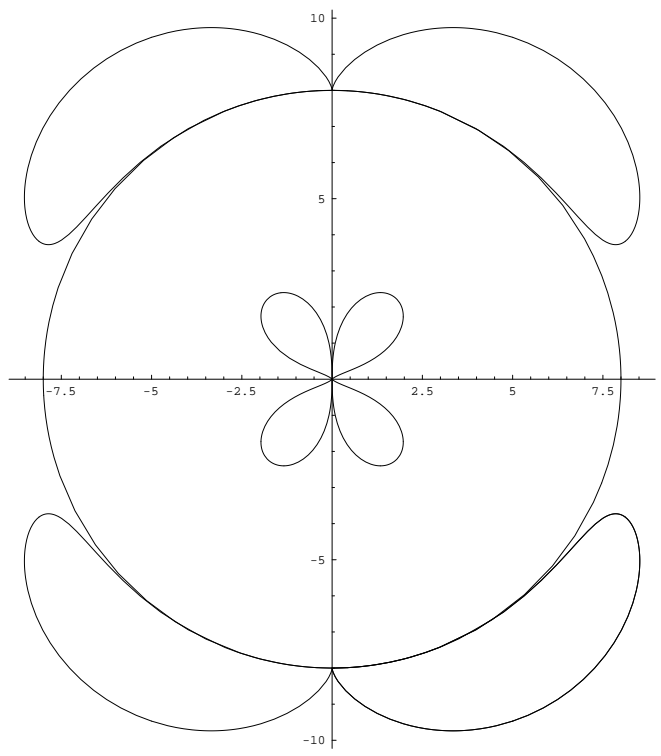

Figure 10: $q_{1}=1, r_{e h}=8, r_{c h}=0$. 